\title{
Global strong solutions and large time behavior of 2D tropical climate model with zero thermal diffusion
}

\author{
Dongjuan $\mathrm{Niu}^{1}$ and Huiru $\mathrm{Wu}^{1}$ \\ ${ }^{1}$ Capital Normal University
}

December 21, 2021

\begin{abstract}
In this article, we study the global well-posedness and large-time behaviors of solutions to the two-dimensional tropical climate system with zero thermal diffusion for a small initial data in the whole space. The main approaches include high and low frequency decomposition method and exploiting the structure of system (1) to obtain the estimates of thermal dissipation. We utilize the time decay properties of the kernels to a linear differential equation to obtain the decay rates of solutions of the low frequency part and the decay property of exponential operator for the high frequency part. The key ingredient here is the explicit large-time decay rate of solutions.
\end{abstract}

\section{Hosted file}

toSubmitMMAS (1).pdf available at https://authorea.com/users/397856/articles/550174-globalstrong-solutions-and-large-time-behavior-of-2d-tropical-climate-model-with-zero-thermaldiffusion 